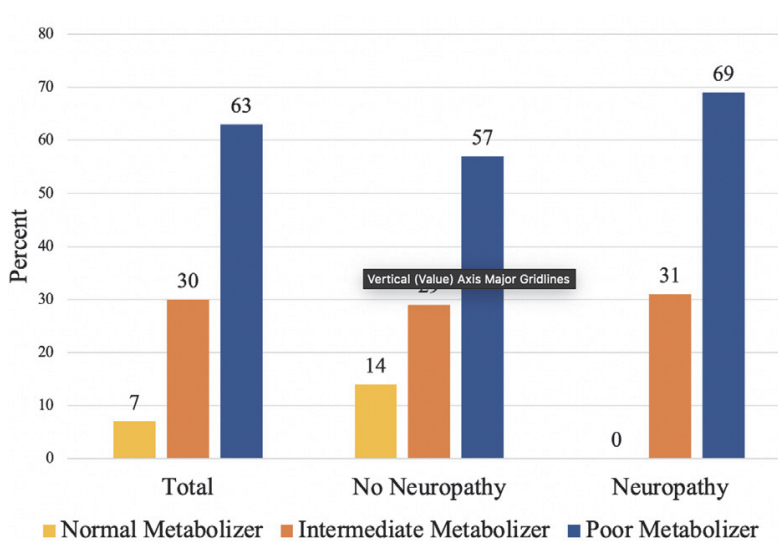

Abstract 736 Figure 1 Differences in CYP3A5 phenotype compared between all patients, those without CIPN, and those with CIPN CYP3A5 Genotype

$(\mathrm{p}=0.023)$, CYP3A5 phenotype $(0.021)$, and CYP2D6 genotype $(p=0.009)$ were associated with increased risk of CIPN. Patients who developed CIPN and had CYP3A5 genomic alterations most often were categorized as having the $3 / 3$ allele (63\%) and were also categorized as poor metabolizers (69\%). Among the 38 genotypes of CYP2D6 there was no variation which contained the majority of patients.

Conclusion* Pharmacogenomics appear associated with the development of CIPN and maybe able to help personalize treatment decision making. This data supports future largescale trials to better determine and define molecular variants which lead to CIPN.

\section{SHORT- AND LONG-TERM UROLOGICAL COMPLICATIONS AFTER SURGERY FOR EARLY-STAGE CERVICAL CANCER}

MT Marina Martín*, M Ivañez Muñoz, P Padilla Iserte, V Lago, MT Luis Javier, M Gurrea, B Segarra Vidal, S Domingo. University Hospital La Fe, Gynecologic Oncology, Valencia, Spain

\subsection{6/ijgc-2021-ESGO.579}

Introduction/Background* To determine the incidence of shortand long-term urological complications after surgery in patients with cervical cancer.

Methodology We performed a review involving women who underwent radical hysterectomy or trachelectomy for earlystage cervical cancer during the 2011-2020 period at University Hospital La Fe. Women diagnosed with locally advanced

\section{Abstract 871 Table 1 Baseline characteristics}

\begin{tabular}{lll}
\hline Characteristics & $\begin{array}{l}\mathrm{n}=96 \\
\text { Rate } \\
(\%)\end{array}$ \\
\hline Age (years) [mean \pm SD] & $49.1 \pm 21,7$ \\
Body mass index BMI [median (range)] & $26.5(17.3-50)$ \\
FIG0 stage (2009) & & \\
- IA1/IA2 & 8 & 8.3 \\
- IB1 & 71 & 74 \\
- IB2 & 7 & 7.3 \\
- IIA1 & 9 & 9.4 \\
- IIB & 1 & 1
\end{tabular}

FIGO stage (2018)

- IA1/1A2

$-\mid \mathrm{B} 1$

- IB2

- IB3

- IIA1

- IIB

Histological type

- Squamous

- Adenocarcinoma

- Adeno-squamous

- Others $\left(^{*}\right)$

Type of surgery

- Radical hysterectomy

- Radical trachelectomy

- Other: radical colpectomy

Surgical approach

- LPS

- LPT

- Vaginal

SLN

- Performed

- Not performed

Surgical time (min) [mean \pm SD]

Final FIGO stage (2009)

- IA1/1A2

$-\mid \mathrm{B} 1$

- IB2

- IIA1

- IIA2

- IIB

Final FIGO stage (2018)

- IA1/1A2

- IB1

- IB2

- IB3

- IIA1

- IIA2

- IIB

- IIIC1

- IIIC2

Intraoperative complications

8.3

$25 \quad 26$

$41 \quad 42.7$

$10 \quad 10.4$

$8 \quad 8.3$

11

$53 \quad 55.2$

$35 \quad 36.5$

$2 \quad 2.1$

$6 \quad 6.25$

$\begin{array}{ll}89 & 92.7\end{array}$

$6 \quad 6.3$

$75 \quad 78.1$

$15 \quad 15.6$

$6 \quad 6.3$

$67 \quad 69.8$

$248.3 \pm 56.8$

$9 \quad 9.4$

$69 \quad 71.9$

$9 \quad 9.4$

$5 \quad 5.2$

11

3.1

- Bowel injury

- Urological injury

- Vascular injury

- Nerve injury

- Others

Short-term postoperative complications ( $<30$ days) (Clavien-dindo)

- II

- III

- IV

- V

Medium/long-term postoperative complications (>30 days) (Clavien

dindo)

- II

- III

- IV

$-\mathrm{V}$

Hospital stay $[$ mean \pm SD]

Mortality (30 days)

(*) Others: small cell neuroendocrine carcinoma, lymphoepithelioma type carcinoma, clea cell carcinoma, adenosarcoma with sarcomatous growth and "glassy cell" adenocarcinoma variant) 


\begin{tabular}{|c|c|c|}
\hline Characteristics & $n=96$ & $\begin{array}{l}\text { Rate } \\
(\%)\end{array}$ \\
\hline Preoperative ureteral stent & 4 & 4.2 \\
\hline \multicolumn{3}{|l|}{ Long-term postoperative complications type ( $>30$ days) } \\
\hline - Urinary & 24 & 27.3 \\
\hline - Bowel/abdominal & 2 & 2.3 \\
\hline - Pulmonary & 0 & 0 \\
\hline - Infectious & 2 & 2.3 \\
\hline - Venous thromboembolism & 2 & 2.3 \\
\hline - Others & 21 & 23.9 \\
\hline \multicolumn{3}{|l|}{ Urological complications } \\
\hline - Infection & 16 & 18.2 \\
\hline - Ureteral obstruction/stenosis & 4 & 4.5 \\
\hline - Fistula (ureterovaginal/vesical) & 5 & 5.7 \\
\hline - Bladder injury & 1 & 1.1 \\
\hline $\begin{array}{l}\text { - Urological dysfunction (hypocontractility, incontinence, low- } \\
\text { compliance bladder) }\end{array}$ & 5 & 5.7 \\
\hline - Urinary sepsis & 2 & 2.3 \\
\hline - Haematuria & 0 & 0 \\
\hline - Acute urinary retention & 0 & 0 \\
\hline CT-urography performed & 14 & 15.9 \\
\hline \multicolumn{3}{|l|}{ Urological complications treatment } \\
\hline \multicolumn{3}{|l|}{ - Ureteral stent } \\
\hline o Unilateral & 5 & 5.7 \\
\hline o Bilateral & 5 & 5.7 \\
\hline - Suprapubic catheter & 2 & 2.3 \\
\hline \multicolumn{3}{|l|}{ - Nephrostomy } \\
\hline o Unilateral & 1 & 1.1 \\
\hline o Bilateral & 0 & 0 \\
\hline - Re-surgery & 5 & 5.7 \\
\hline o Fistula repair & 2 & 2.3 \\
\hline o Ureteral reimplantation & 2 & 2.3 \\
\hline o Fistula repair + Ureteral reimplantation & 1 & 1.1 \\
\hline o Nephrectomy & 1 & 1.1 \\
\hline
\end{tabular}

Abstract 871 Table 3 Oncological outcome

\begin{tabular}{lll}
\hline Characteristics & $\mathrm{n}=96$ & $\begin{array}{l}\text { Rate } \\
(\%)\end{array}$ \\
\hline Adjuvant treatment & 55 & 62.5 \\
- No & 17 & 19.3 \\
- RT & 17 & 19.3 \\
- RT/CT & 8 & 9.1 \\
Recurrence & & \\
Recurrence location & 2 & 2.1 \\
- Local & 1 & 1 \\
- Regional & 1 & 1 \\
- Locoregional & 1 & 1 \\
- PALN & 3 & 3.1 \\
- Distant and & & \\
multisite & 5 & 5.2 \\
Death of disease & $82.1 \%$ & \\
5-years DFS & $93.4 \%$ & \\
5-years OS &
\end{tabular}

CT=chemotherapy; DFS=disease free survival; OS=overall survival; PALN= para-aortic lymph nodes; $\mathrm{RT}=$ radiotheraoy. cervical cancer, patients with positive sentinel lymph node (SLN) in the intraoperative study and those with persistent or recurrent disease were excluded.

Result(s)* A total of 96 patients underwent surgery for earlystage cervical cancer. The pre-surgical stage was: IA $8.3 \%$, IB1 74\%, IB2 7.3\%, IIA1 9.4\%, IIB 1\%. Radical surgery was performed in 89 patients, fertility preserving surgery in 6 patients and radical colpectomy in 1 patient. Surgery approach was laparotomy, laparoscopy and vaginally in $15.6 \%, 78.1 \%$ and $6.3 \%$ of women, respectively. SLN biopsy was performed in $69.8 \%$ of patients. The mean operating time was 248.3 minutes and the mean hospital stay was 3.4 days.

The intraoperative and immediate postoperative complication rate $(<30$ days $)$ was $12.5 \%$ and $27 \%$ respectively. The Clavien-Dindo classification: II (61.5\%), III (38.5\%), IV and V $(0 \%)$. No statistical differences were found according to surgical approach and type of surgery in intraoperative $(p=1.00$; $\mathrm{p}=0.47)$ nor short-term complications $(\mathrm{p}=1.00 ; \mathrm{p}=0.5)$. Longterm complication rate (>30days) was $10.4 \%$. No differences were found according to surgical approach and type of surgery $(\mathrm{p}=0.28 ; \mathrm{p}=0.5)$. Urological complications occurred in 20 patients (20.8\%). Nine of them required ureteral catheter placement, 5 patients required re-intervention and 1 patient a nephrostomy.

Nearly $40 \%$ of the patients received adjuvant treatment: $58 \%$ only radiotherapy and $42 \%$ radiotherapy + chemotherapy. Six patients (6.25\%) presented urinary complications after $\mathrm{RT}$ and four patients $(4.2 \%)$ presented long-term urinary complications. No statistical differences in urological complications were found according to RT treatment $(\mathrm{p}=0.64)$.

The mean follow-up time was 39.7 months. Eleven patients $(11.5 \%)$ presented recurrence of their tumour process. Disease-free survival and overall survival at 5 years were $82.1 \%$ and $93.4 \%$ respectively

Conclusion* Surgery for early cervical cancer leads to urological complications in a considerable percentage of patients, however most of which are mild and self-limiting with medical treatment. Only a small group of patients will experience serious complications in the medium to long term.

\section{ADVANCED GYNECOLOGICAL CANCER: QUALITY OF LIFE ONE YEAR AFTER DIAGNOSIS}

B Jónsdóttir* , A Wikman, I Sundström Poromaa, K Stålberg. Uppsala University, Women's and Children's Health

\subsection{6/ijgc-2021-ESG0.580}

Introduction/Background* Gynecological cancer treatment can include various types of surgery, radiation and/or chemotherapy. It can leave irreversible changes on women's physical health and influence their psychological wellbeing affecting their quality of life. The aim of this study is to examine the quality of life of women with advanced gynecological cancer one year after diagnosis and identify possible predictive factors.

Methodology Women with endometrial, ovarian or cervical cancer included in the U-CAN (Uppsala-Umeå Comprehensive Cancer Consortium) database in years 2012-2019 were applicable for the study. Data from a general health questionnaire and the 36-item Short Form Survey (SF-36) were gathered along with information from medical records on histology, 